\title{
Functional Properties of Human-Derived Mesenchymal Stem Cell Spheroids: A Meta-Analysis and Systematic Review
}

\author{
Sarah Ezquerra, ${ }^{1}$ Amparo Zuleta, ${ }^{1}$ Rodrigo Arancibia, ${ }^{1,2}$ José Estay, ${ }^{1,2}$ \\ Francisco Aulestia $\left(\mathbb{D},{ }^{1,2} \text { and Flavio Carrion }{ }^{3}\right)^{3}$ \\ ${ }^{1}$ Cellus Medicina Regenerativa S.A, Complejo Boulevard Kennedy, Av. Presidente Kennedy, 5741 Santiago, Chile \\ ${ }^{2}$ Cellus Biomédica, Parque Tecnológico de León, C/ Julia Morros s/n, 24009, León, Spain \\ ${ }^{3}$ Programa de Inmunología Traslacional, Facultad de Medicina, Clínica Alemana Universidad del Desarrollo, \\ Santiago 8320000, Chile \\ Correspondence should be addressed to Francisco Aulestia; francisco.aulestia@cellus.cl \\ and Flavio Carrion; flavio.carrion@gmail.com
}

Received 23 August 2020; Revised 31 January 2021; Accepted 12 February 2021; Published 5 April 2021

Academic Editor: Mustapha Najimi

Copyright ( 2021 Sarah Ezquerra et al. This is an open access article distributed under the Creative Commons Attribution License, which permits unrestricted use, distribution, and reproduction in any medium, provided the original work is properly cited.

\begin{abstract}
Mesenchymal stem cells (MSC) are adult multi-potent cells that can be isolated from many types of tissues including adipose tissue, bone marrow, and umbilical cord. They show great potential for cell therapy-based treatments, which is why they are being used in numerous clinical trials for a wide range of diseases. However, the success of placebo-controlled clinical trials has been limited, so new ways of improving the therapeutic effects of MSC are being developed, such as their assembly in a 3D conformation. In this meta-analysis, we review aggregate formation, in vitro functional properties and in vivo therapeutic potential displayed by adipose tissue, bone marrow, and umbilical cord-derived MSC, assembled as spheroids. The databases PubMed and SciELO were used to find eligible articles, using free-words and MeSH terms related to the subject, finding 28 published articles meeting all inclusion and exclusion criteria. Of the articles selected 15 corresponded to studies using MSC derived from bone marrow, 10 from adipose tissue and 3 from umbilical cord blood or tissue. The MSC spheroids properties analyzed that displayed enhancement in comparison with monolayer 2D culture, are stemness, angiogenesis, differentiation potential, cytokine secretion, paracrine and immunomodulatory effects. Overall studies reveal that the application of MSC spheroids in vivo enhanced therapeutic effects. For instance, research exhibited reduced inflammation, faster wound healing, and closure, functional recovery and tissue repair due to immunomodulatory effects, better MSC engraftment in damaged tissue, higher MSC survival and less apoptosis at the injury. Still, further research and clinical studies with controlled and consistent results are needed to see the real therapeutic efficacy of MSC spheroids.
\end{abstract}

\section{Introduction}

Mesenchymal stem cells (MSC) are plastic adherent, fibroblast-like, non-hematopoietic progenitor cells isolated from a variety of adult tissues. MSC have self-renewal capabilities, and they can differentiate into several tissue-specific lineages including osteoblasts, chondrocytes, adipocytes, hepatocytes and cardiomyocytes among others [1, 2]. MSC can be harvested from several tissues, including bone-marrow (BM-MSC), adipose tissue (AT-MSC), umbilical cord (UCMSC), Wharton's jelly (WJ-MSC), gingiva (G-MSC) and cartilage tissue (C-MSC), among others [3, 4]. The International
Society for Cellular Therapy (ISCT) establishes three key features to identify MSC: First, mesenchymal stem cells must display adherence to plastic when cultured under standard conditions. Second, MSC population must express CD105, CD73 and CD90 $(\geq 95 \%)$ and not express CD45, CD34, HLA-DR, CD14 or CD11b, CD79a or CD19 $(\leq 2 \%)$. Third, MSC cultured in vitro must show osteogenic, adipogenic and chondrogenic differentiation [5]. Besides their ability for multipotent differentiation, MSC are mainly considered as immune evasive cells and they secrete many key trophic factors contributing to tissue repair and regeneration [6-8]. Therefore, MSC appear to be an appealing alternative of 
treatment in regenerative medicine because it is possible to administrate allogenic populations of these cells or more differentiated lineages [9]. Based on MSC capacity to differentiate and mature into specific phenotypes, their immunomodulatory properties, and their distinct migratory and potent trophic effects during tissue regeneration, the potential for clinical applications is remarkable $[10,11]$.

At the time of writing this review, there are currently 874 clinical studies reported using MSC to treat different diseases (www.clinicaltrials.gov). However, the beneficial effects of MSC based therapies in small clinical studies are often not substantiated by large randomized double blind, placebocontrolled clinical trials $[12,13]$. Several clinical studies, unable to meet the clinical goal, suggest that after prolonged ex vivo expansion of BM-MSC, their immune-suppressive properties change and display deficient survival rate posttransplantation $[14,15]$. This implies that it is necessary to comprehend the regenerative and immunomodulatory mechanisms by which MSC exert their action. New ways of enhancing the functional properties of MSC are also needed, which until now have only been cultivated in monolayer 2D culture for clinical applications. Although a simple procedure, some in vivo essential qualities and traits are compromised or lost.

Therefore, 3D cell culture emerges as a new therapeutic alternative, offering better preservation of those features, as allowing a better mimicking of in vivo conditions, particularly cells self-assembling, as observed during embryonic development, thus increasing cells interactions $[16,17]$. To promote this interaction, several spheroid formation techniques have been developed. Contrary to monolayer culture, three-dimensional culture of MSC as spheroids causes considerable changes in the gene expression pattern $[18,19]$. Diverse studies propose that the functionality of stem cells can be improved and unsuitable migration of cells, after injection in the target tissue, can be avoided, by aggregate formation [20-22]. However, the exact mechanism involved in $3 \mathrm{D}$ conformation is still unknown, although several signalling pathways have been proposed [23-28]. For example, it has been described that decreased expression of transcriptional co-activators yes-associated protein/transcriptional co-activator with PDZ-binding motif (YAP/TAZ) in MSC cultured in $3 \mathrm{D}$ conformation, was associated with a loss of the actin cytoskeleton $[27,28]$. In other study, Zhang et al., showed an increase in the expression of hypoxia-inducible factor (HIF)-1 and $-2 \alpha$ in MSC spheroids which was related to increased resistance to apoptosis triggered by oxidative stress [23]. This meta-analysis was conducted to review spheroid formation, in vitro functional properties and in vivo therapeutic potential displayed by adipose tissue, bone marrow, and umbilical cord-derived MSC, assembled as spheroids.

\section{Materials and Methods}

2.1. Search Strategy. The following databases, PubMed and SciELO, were searched for eligible published articles until May 2019, using specific free-words and MeSH terms. Different combination of the following terms were used: type of cell: mesenchymal, stromal, stem cell, pluripotent, multipotent; cell organization: 3D, spheroid, cluster, organoid, aggregates; application: cell therapy, tissue regeneration, treatment, therapy, functional recovery; pathologies: skeletal muscle, muscle cartilage, tendon or joint pathologies; osteoarthritis, chronic injuries, immune diseases, neurodegenerative diseases, neurological pathology, bone pathology; culture conditions: hypoxia, low oxygen, xeno-free, serumfree, animal-free; properties: secretome, exosome, vesicles, immunoregulatory. Of the results obtained only articles published in English were included and, articles related to cancer and published earlier than 2008 were excluded. Other potential articles were identified from references within the selected articles or reviews related to the topic.

2.2. Selection Criteria. Inclusion criteria are listed as: a) Human MSC; b) source of MSC either bone marrow, adipose tissue or umbilical cord; c) studies focused on cell therapy and regenerative medicine. Exclusion criteria are listed as a) article does not meet inclusion criteria; b) reviews and case reports; c) work focused on application and/or use of MSC spheroids in bioengineering.

2.3. Data Extraction. The data extracted from included articles consisted of: authors, year, country, title, MSC tissue source, cell aggregation protocol, culture conditions, spheroid measurements, functional properties in vitro, altered markers for said properties, therapeutic effects in vivo and study model used.

2.4. Limitations. Only articles published in English were included, which may leave out other eligible publications that were reported in other languages. Therefore, the results should be interpreted cautiously due to the limited data.

\section{Results and Discussion}

3.1. Search Results. After a screening of 254 articles identified by searching in PubMed $(\mathrm{n}=131)$ and SciELO $(\mathrm{n}=123)$ associated with MSC in 3D conformation, only 71 articles were assessed for eligibility according to the search strategy described in materials \& methods. Of these 71 articles, only 28 articles were incorporated in this meta-analysis according to the inclusion criteria described in materials \& methods (human MSC, MSC harvested from bone marrow, adipose tissue or umbilical cord, and studies focused on cell therapy and regenerative medicine (Figure 1).

The list of the 28 articles chosen and their basic description (author(s), year, country, source of MSC, properties and parameters evaluated, and ref number) is shown in Table 1.

It can be noted that the earliest work included in this review is from May 2010 [29] and the latest being published in April 2019 [30]. Most articles are published in $2017(n=5)$, succeeded by 2014, 2016 and 2018, each with 4 articles. The most articles collected are from work conducted in the USA with a total of 13 published articles comprising a $46.4 \%$ of all articles included, followed by South Korea with a total of $5(17.9 \%)$ and China with $3(10.7 \%)$ (Table 1). Of the 28 articles selected 15 corresponded to studies using MSC derived 


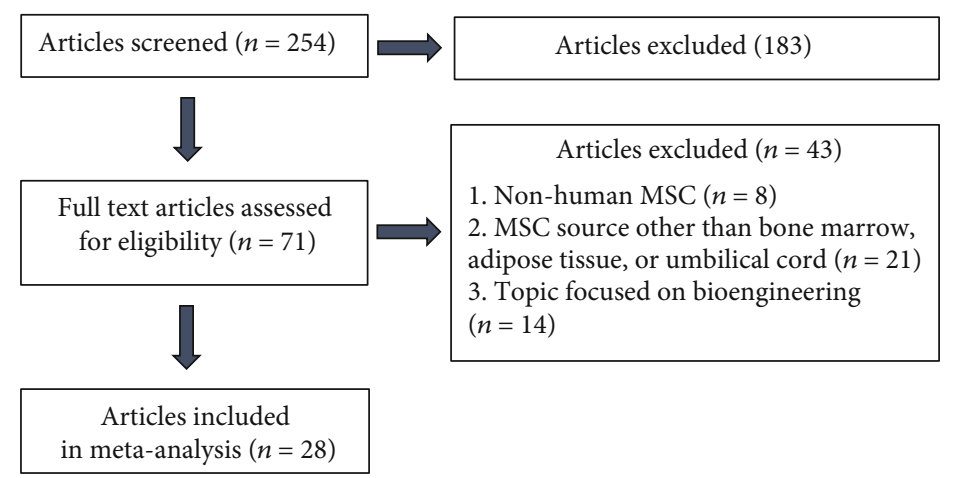

Figure 1: Meta-Analysis Study Selection.

from bone marrow, 10 from adipose tissue and 3 from umbilical cord blood or tissue.

In all, the most evaluated characteristics of MSC spheroids are stemness, angiogenesis, differentiation, cytokine secretion, paracrine effect, metabolic function, and immunomodulatory effects (Table 1).

3.2. Cell Aggregation Protocol. There are several methods and techniques to form MSC spheroids, here we have found that the most used procedure is the hanging drop method $(n=13)$ [19-21, 29, 31-39], followed by forced-aggregation $(n=4)$ [40-43], low attachment $(\mathrm{n}=4)$ [44-47], spontaneous assembly $(n=4)[30,48-50]$, then chitosan films $(n=2)[18,51]$, and finally hyaluronic acid gel $(n=1)$ [52] (Figure 2). The hanging drop technique consists of plating the MSC suspension in droplets of determined volume on the lid of a culture dish. The lid is turned over in a swift and careful move and placed on top of the culture plate which is filled with a solution to avoid drop evaporation. The spheroid is formed in the apex of the drop. This method can yield spheroids of controlled sizes, determined by the number of cells in each drop associated with the concentration of the cell suspension and volume of droplet, and there is no need for specialized and expensive equipment $[32,38]$. Even though this technique shows many advantages it still poses a problem for large scale production of MSC spheroids for therapeutic applications $[53,54]$. Other of the techniques used for the formation of MSC spheroids is the forced aggregation method which consists in applied centrifugal forces to induce MSC in vitro aggregation using micro-well plates in presence or not of biomaterials [40, 42, 43]. On the other hand, it has been showed that immunomodulatory activity of MSC does not seem to be spontaneous but requires MSC to be 'licensed' by inflammatory microenvironment to exert their effects $[11,55]$. In this line, Krampera and Ren demonstrated that MSC-mediated immunosuppression requires preliminary activation of the MSC by immune cells through the secretion of the proinflammatory cytokine IFN-g, alone or together with TNFa, IL-1a or IL-1b [56, 57]. In this review, 4 of 28 articles selected, use cytokine priming to improve the functional properties of MSC (Figure 2) [29, 42-44].

3.3. Culture Conditions. For MSC spheroids to be eligible for clinical applications they need to be xeno-free (not contain- ing any animal-derived components). Serum containing media can carry unexpected agents risking viral or mycoplasma contamination [48]. Then, spheroid formation in media without FBS (fetal bovine serum) is critical. In this analysis, we identified that 8 of the 28 articles included, used serum-free media in their cell aggregation protocols (Figure 2). The importance of FBS is highly noted for cell aggregation during spheroid formation, correlated with spheroids exhibiting faster assembly and more defined edges. Some of the strategies used to substitute FBS include using chemically defined media, in which all components are known, and composition can be controlled or supplementing media with patient-derived serum or human serum albumin [39].

Also controlling the oxygen level during spheroid assembly can have beneficial effects. Hypoxia as a priming method of MSC aggregates was used in three articles [30, 33, 46] (Figure 2). Some research has pointed out that hypoxic conditions during cell aggregation can improve MSC properties. For example, the enhancement of the paracrine effect due to higher levels of IL-6, IL-8, and MCP-1 [46]. From a physiological point of view, MSC cultured under hypoxic conditions can better prepare cells for an ischemic environment typical of damaged tissue.

3.4. Spheroid Diameter. Surprisingly, there does not seem to be homogeneity of spheroid size, 6 articles show a similar diameter range of $200-500 \mu \mathrm{m}, 4$ articles a diameter range between $100-200 \mu \mathrm{m}, 3$ a diameter between $0-50 \mu \mathrm{m}, 1$ has spheroids with diameter between 50-100 $\mu \mathrm{m}$ and finally 1 article with spheroid diameter $>500 \mu \mathrm{m}$ (Figure 2). Another measurement used to describe spheroid size is the number of cells within spheroids, and 5 articles displayed spheroids with 10,000-25,000 cells/spheroids, 3 with 200-1,000 cells/ spheroids and 1 with cell concentration greater than $>25,000$ cells/spheroids (Figure 2). This is the initial number of cells at the beginning of the aggregation process. Some articles $(n=4)$ made no mention of MSC spheroid diameter or size (Figure 2). Spheroid size is important because the diameter can determine nutrient and oxygen availability, as well as mechanical forces created by the cell-to-cell contacts between MSC, modulating gene expression [58].

3.5. In Vitro Properties of MSC Spheroids. Different approaches used to assess the functional properties of MSC 


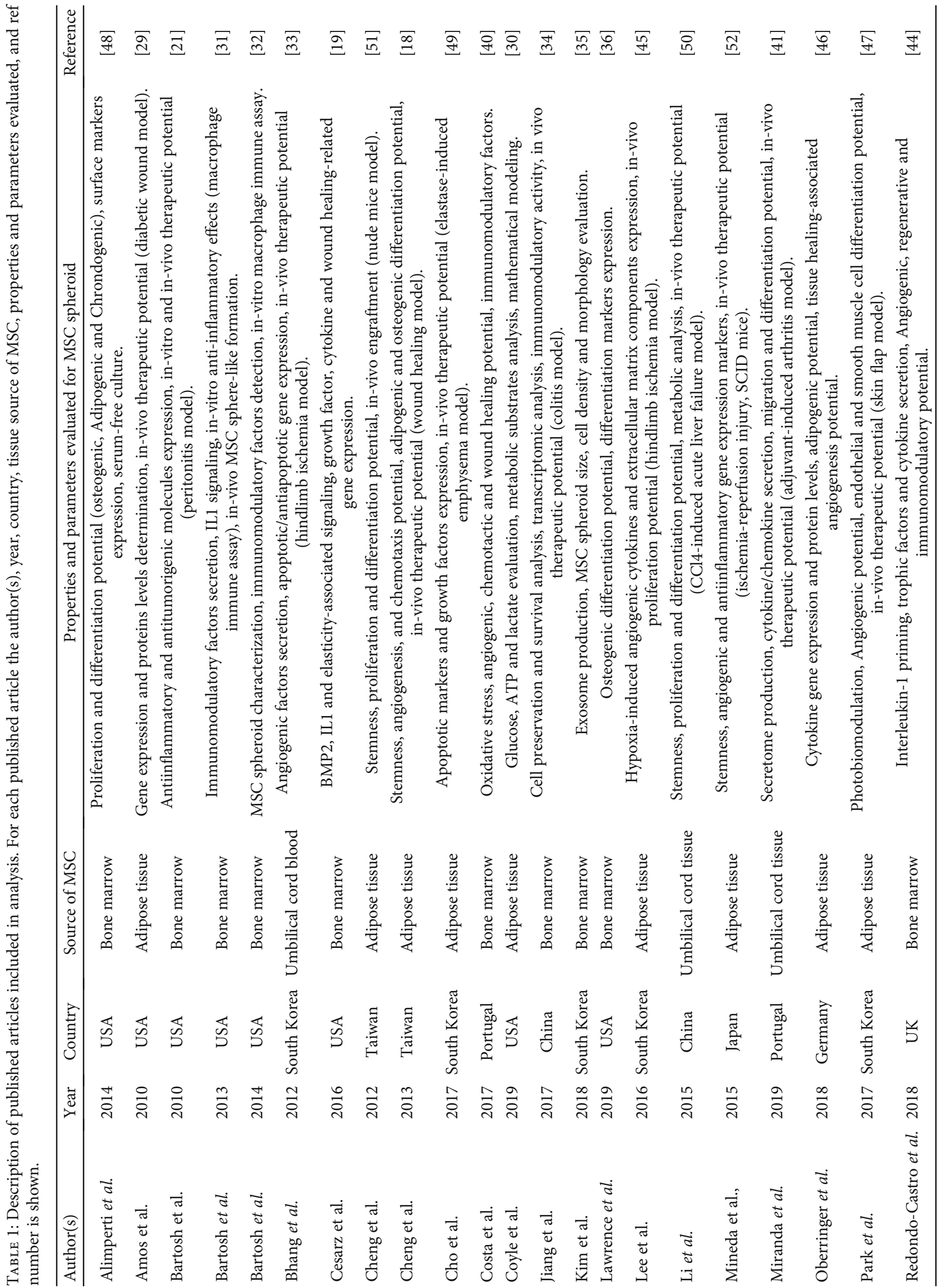




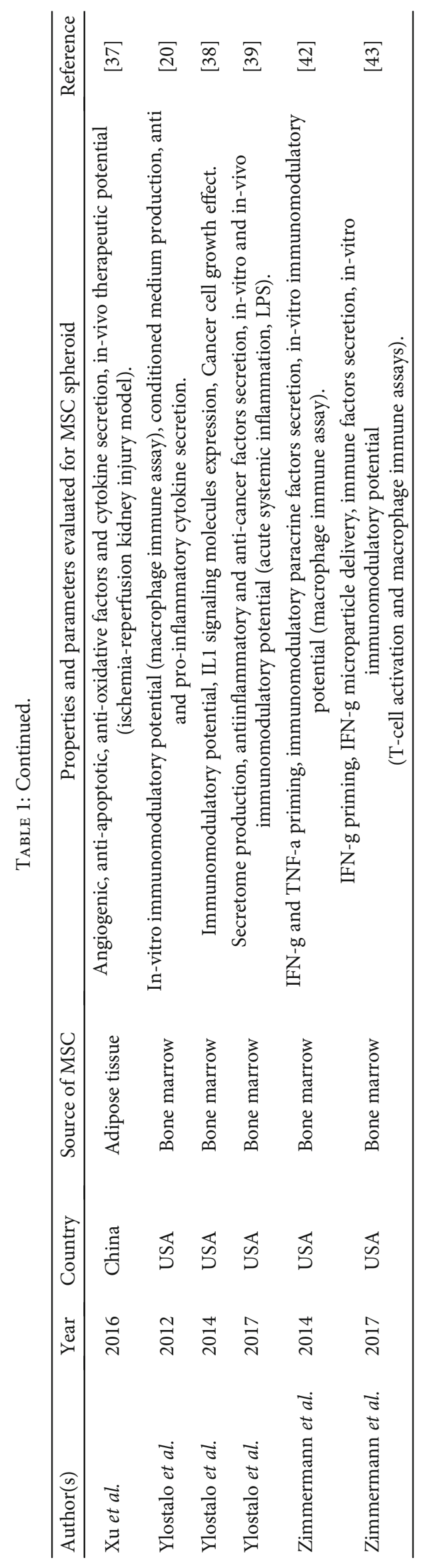




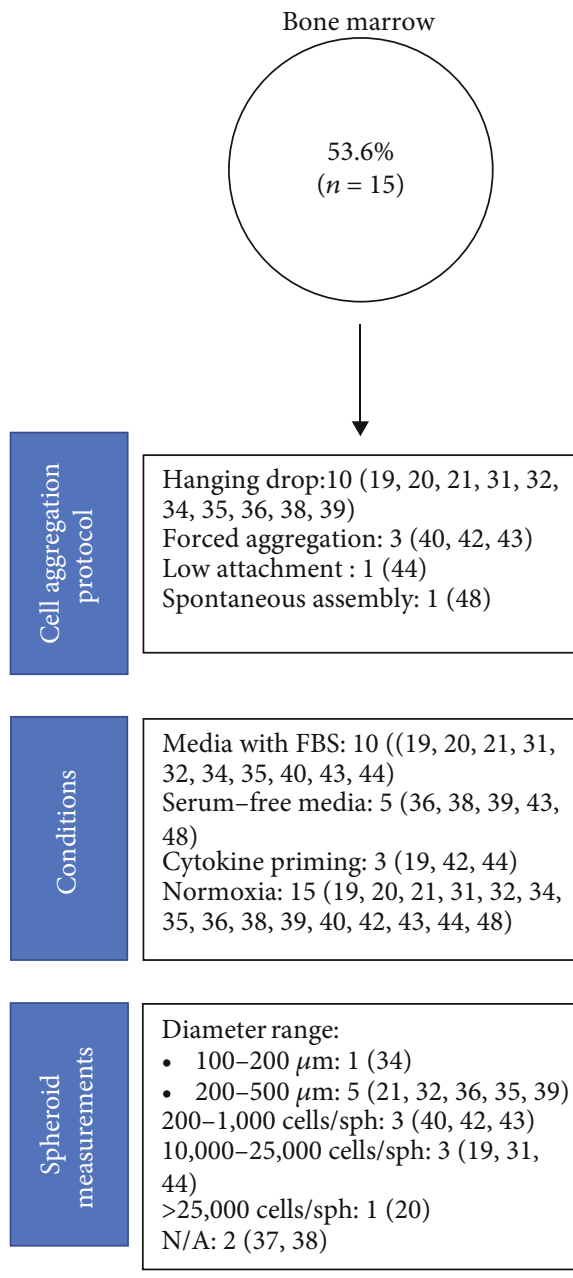

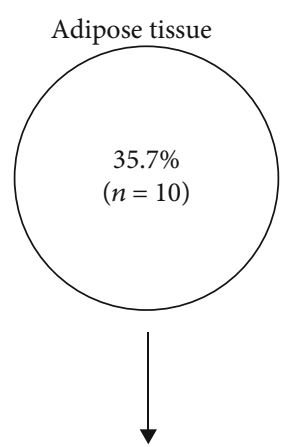

Hanging drop: $2(29,37)$

Low attachment: $3(45,46,47)$

Spontaneous assembly: $2(30,49)$

Chitosan films: $2(18,51)$

Hyaluronic acid gel: 1 (52)

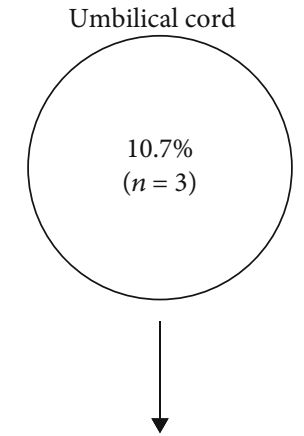

Media with FBS: 8 (18, 30, 37, 45,

$46,47,51,52$ )

Serum-free media: $2(29,49)$

Cytokine priming: 1 (29)

Normoxia: $8(18,29,37,45,47,49$,

$51,52)$

Hypoxia: $2(30,46)$

\section{Diameter range:}

- 0-50 $\mu \mathrm{m}: 2(45,52)$

- 100-200 $\mu \mathrm{m}: 3(29,30,51)$

Diameter $>500 \mu \mathrm{m}: 1(47)$

10,000-25,000 cells/sph: $2(46,37)$

N/A: $2(18,35)$
Hanging drop: 1 (33)

Forced aggregation: 1 (41)

Spontaneous assembly: 1 (50)

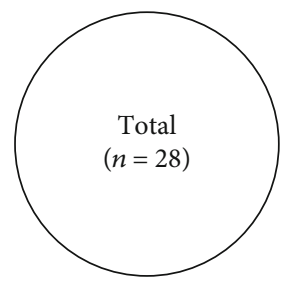

Total

Hanging drop: 13

Forced aggregation: 4

Low attachment: 4

Spontaneous assembly: 4

Chitosan films: 2

Hyaluronic acid gel: 1

Media with FBS: $2(33,41)$

Serum-free media: 1 (50)

Normoxia: $2(50,41)$

Hypoxia: 1 (33)

Media with FBS: 20

Serum-free media: 8

Cytokine priming: 4

Normoxia: 25

Hypoxia: 3

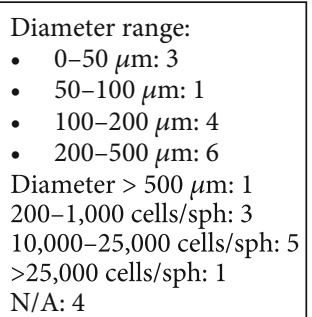

FIgure 2: Cell aggregation protocols, culture conditions, and spheroid diameter for selected articles by source of MSC. Article reference number in parenthesis.

can be identified from the selected works. These traits can be studied by variations in gene expression, protein secretion, surface marker expression, culture, and differential staining. Stemness is usually evaluated by the expression of transcription factors Nanog, Oct-3/4, Sox-2, Klf4, c-Myc, and STAT3, but also the expression of surface markers like CD105, CD90, CD73, and CD34 among others [16, 18, 50]. The effect of MSC over angiogenesis, the development of new vessels, is assessed mainly by the expression of the factors VEGF and HGF, among others (Table 2). Differentiation potential to several types of tissue is also studied as a characteristic trait of MSC, generally by different staining techniques like Alizarin Red, Alcian Blue, and Oil Red $\mathrm{O}$ staining, to determine osteogenic, chondrogenic and adipogenic differentiation, respectively. Cytokines and soluble factors secreted by MSC has been attributed to great therapeutic effects. Among the cytokines analyzed are growth factors, chemokines, interleukins, and more (Tables 1 and 2). There is also evidence of the paracrine anti-cancer, antiapoptotic and resistance to oxidative stress of MSC (Tables 1 and 2). There seems to be a great focus on immunomodulatory effects of MSC, so the expression of TSG-6,
PGE2, LIF, IDO, STC1, IL-6, IL-8 and more, is normally evaluated, as well as the capacity of MSC to decrease levels of TNF- $\alpha$ (Table 2).

3.6. In Vivo Therapeutic Effects of MSC Spheroids. Of the total pool of articles reviewed, only 13 assessed the therapeutic effects of MSC spheroids in vivo (Table 3), 2 articles used BM-MSC, 8 articles used AT-MSC and 3 articles used UCMSC. Several study models are used to investigate in vivo effects, such as wound healing or pro-inflammatory disease models, but mostly ischemia animal models are used (Table 3). In vivo studies include spheroid transplantation or application of conditioned media derived from spheroid culture, into the target tissue. Overall studies reveal that the application of MSC spheroids in vivo has enhanced therapeutic effects compared to monolayer 2D culture. Studies showed better outcomes for MSC in 3D conformation including reduced inflammation, faster wound healing and closure, functional recovery and tissue repair due to immunomodulatory effects, better MSC engraftment in damaged tissue, higher MSC survival and less apoptosis at injury (Table 3). 


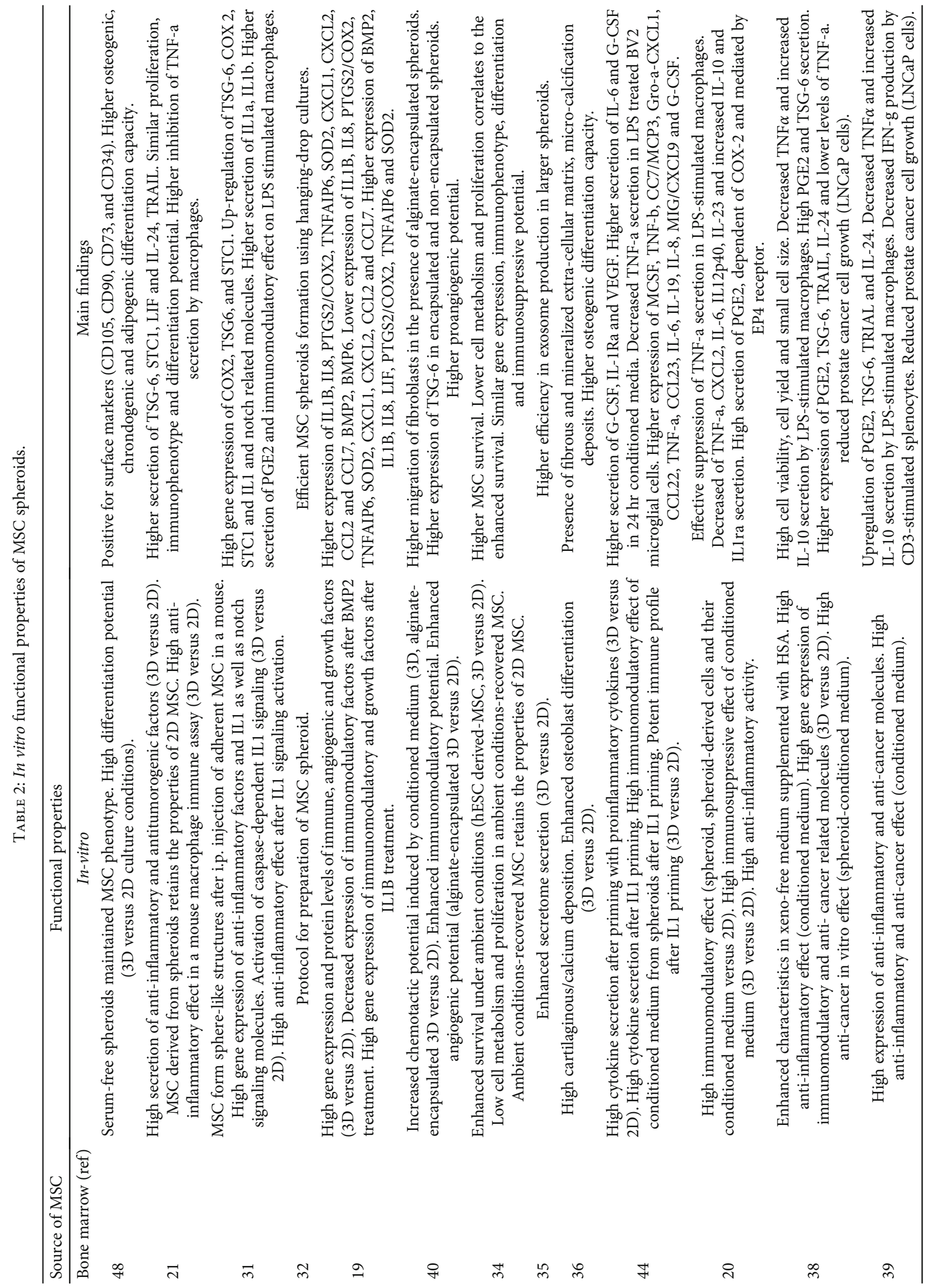




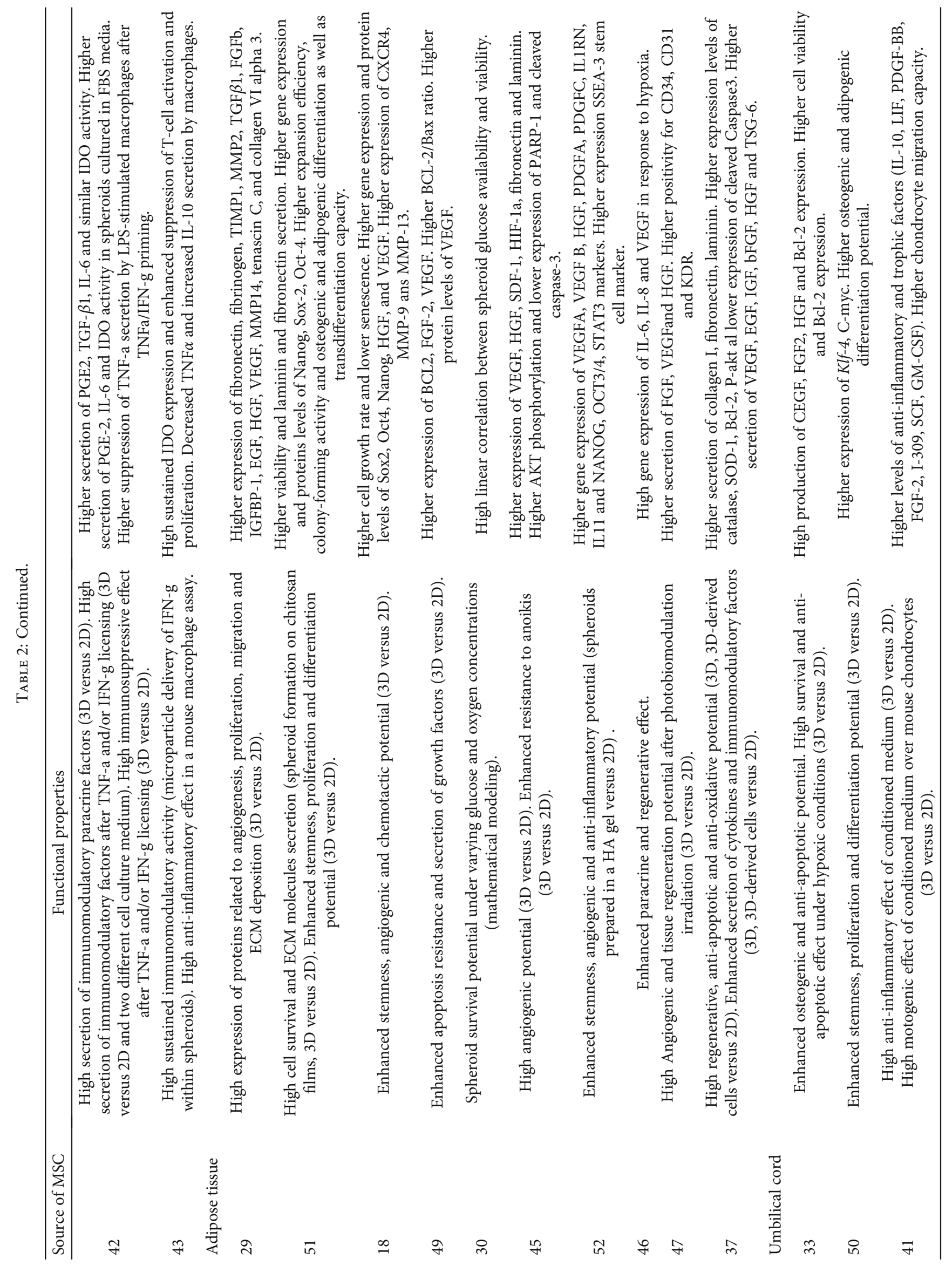




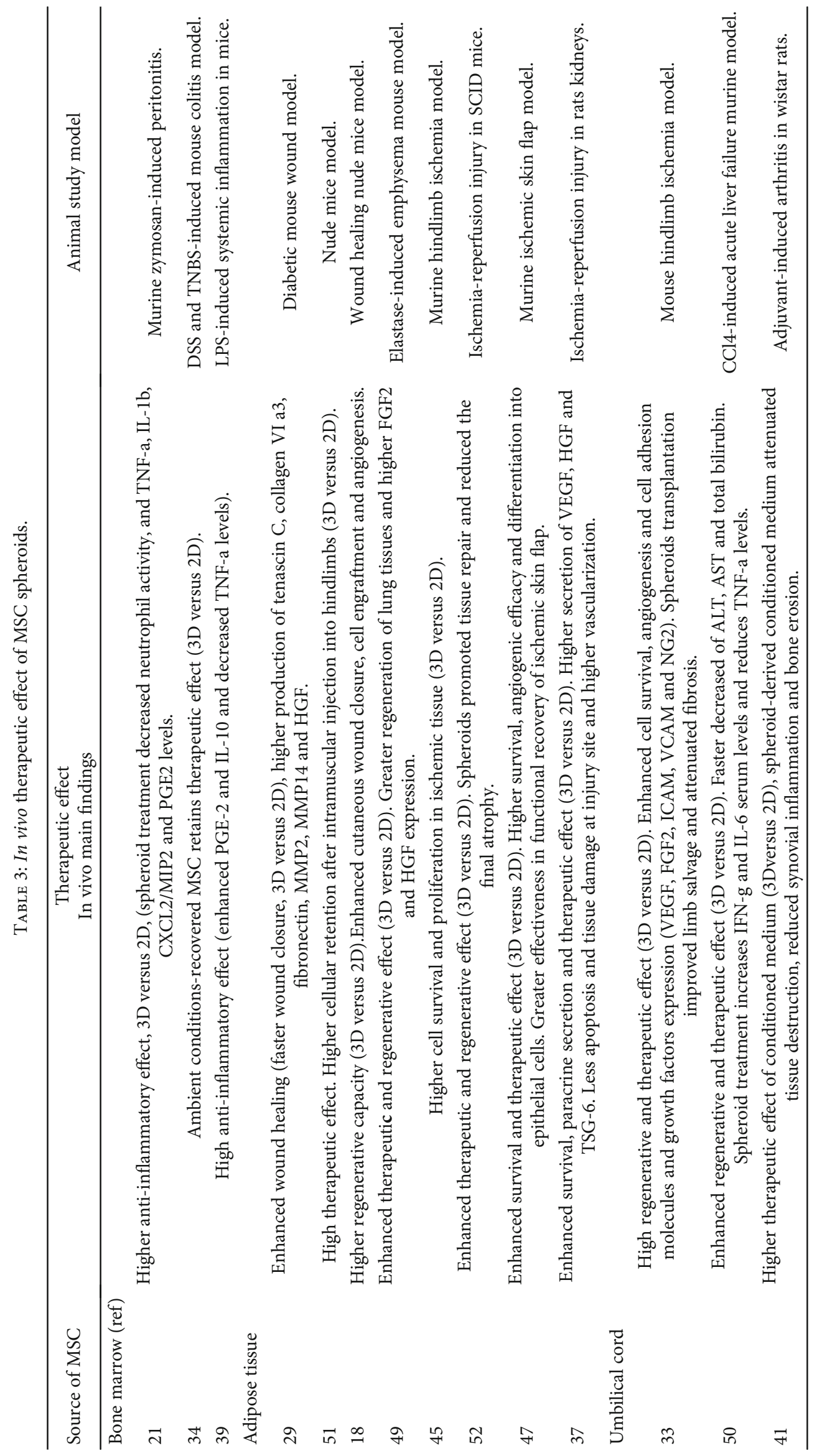


3.7. Limitations of This Study. There are some limitations to this study. First, there is a degree of heterogeneity in the sources of cells and techniques used to produce the MSC spheroids. Second, there are many publications excluded due to technical factors, but still holding conclusions that may be relevant to this research. Finally, there is still a lack of clinical research to test MSC real benefits on a large scale randomized controlled trials.

\section{Conclusion}

Based on the literature reviewed, we can conclude that the most used source of MSC to produce spheroids is bone marrow followed by adipose tissue and umbilical cord. Although MSC can be derived from many adult tissues such as bone marrow, adipose tissue, umbilical cord, and placenta, these sources present some limitations due to differences between donors, extensive in vitro cell culture expansion and clinical trials with inconsistent results $[14,59]$. In this line, MSC derived from human Induced pluripotent stem cells (iPSC) has emerged in the last decade as an excellent therapeutic strategy to overcome the limitations of MSC source, interdonor variability, senescence and culture [60-62]. HumaniPSC-derived MSC possess higher proliferative potential and telomerase activity as well as immunomodulatory and angiogenic properties which has been demonstrated in different preclinical models of disease and clinical trials [61, 63-66]. Interestingly, Ding et al., demonstrated that murine-iPSC-derived MSC cultured in an encapsulated 3D spheroid format display stronger immunomodulation in a murine heart transplantation model [67].

The cell aggregation protocol most used was the hanging drop technique followed by forced-aggregation, low attachment, spontaneous assembly, then chitosan films and hyaluronic acid gel.

The evidence here shows MSC traits are enhanced by 3D culture. Results from the 28 articles selected suggest that MSC display better stemness, angiogenesis, differentiation potential, cytokine secretion, paracrine and immunomodulatory effects when presented as spheroids. At the time of this review, no human trials using MSC spheroids were found at the National Institutes of Health (NIH) website.

In this context, genetically engineering MSC or threedimensional culture, express and secrete important paracrine and immunomodulatory factors such as IDO, PGE2 and TSG-6 suggesting that this might increase the in vivo therapeutic effect of MSC [23, 31, 42, 43].

In comparison with monolayer culture, MSC in $3 \mathrm{D}$ conformation presents as an attractive alternative for therapeutic applications of MSC. However, more studies are needed to evaluate the real therapeutic efficacy of MSC spheroids as well as mechanisms and pathways involved.

\section{Conflicts of Interest}

The authors declare that they have no conflict of interests.

\section{Acknowledgments}

The author would like to thank Anibal Romero at Cellus Medicina Regenerativa and Martin Pendola at NYU College of Dentistry for providing comments that helped improve this manuscript. This work was supported by Corporación de Fomento de la Producción CORFO 17PIDE-80689 to Cellus Medicina Regenerativa S.A and Cellus Biomedica.

\section{References}

[1] M. F. Pittenger, A. M. Mackay, S. C. Beck et al., "Multilineage potential of adult human mesenchymal stem cells," Science, vol. 284, no. 5411, pp. 143-147, 1999.

[2] R. B. Ullah, R. B. Subbarao, and G. J. Rho, "Human mesenchymal stem cells - current trends and future prospective," Bioscience Reports, vol. 35, no. 2, 2015.

[3] L. Da Silva Meirelles, P. C. Chagastelles, and N. B. Nardi, "Mesenchymal stem cells reside in virtually all post-natal organs and tissues," Journal of Cell Science, vol. 119, no. 11, pp. 2204-2213, 2006.

[4] R. Hass, C. Kasper, S. Bohm, and R. Jacobs, "Different populations and sources of human mesenchymal stem cells (MSC): a comparison of adult and neonatal tissue-derived MSC," Cell Communication and Signaling, vol. 9, no. 12, p. 14, 2011.

[5] M. Dominici, K. le Blanc, I. Mueller et al., "Minimal criteria for defining multipotent mesenchymal stromal cells. The International Society for Cellular Therapy position statement," Cytotherapy, vol. 8, no. 4, pp. 315-317, 2006.

[6] J. A. Ankrum, J. F. Ong, and J. M. Karp, "Mesenchymal stem cells: immune evasive, not immune privileged," Nature Biotechnology, vol. 32, no. 3, pp. 252-260, 2014.

[7] F. G. Teixeira and A. J. Salgado, "Mesenchymal stem cells secretome: current trends and future challenges," Neural Regeneration Research, vol. 15, no. 1, pp. 75-77, 2020.

[8] J. Driscoll and T. Patel, "The mesenchymal stem cell secretome as an acellular regenerative therapy for liver disease," Journal of Gastroenterology, vol. 54, no. 9, pp. 763-773, 2019.

[9] Y. Han, X. Li, Y. Zhang, Y. Han, F. Chang, and J. Ding, "Mesenchymal stem cells for regenerative medicine," Cell, vol. 8, no. 8, p. 886, 2019.

[10] F. Figueroa, F. Carrion, S. Villanueva, and M. Khoury, "Mesenchymal stem cell treatment for autoimmune diseases: a critical review," Biological Research, vol. 45, no. 3, pp. 269-277, 2012.

[11] F. Djouad, C. Bouffi, S. Ghannam, D. Noël, and C. Jorgensen, "Mesenchymal stem cells: innovative therapeutic tools for rheumatic diseases," Nature Reviews Rheumatology, vol. 5, no. 7, pp. 392-399, 2009.

[12] J. J. Galipeau, "The mesenchymal stromal cells dilemma-does a negative phase III trial of random donor mesenchymal stromal cells in steroid-resistant graft-versus-host disease represent a death knell or a bump in the road?," Cytotherapy, vol. 15, no. 1, pp. 2-8, 2013.

[13] H. Caplan, S. D. Olson, A. Kumar et al., "Mesenchymal stromal cell therapeutic delivery: translational challenges to clinical application," Frontiers in Immunology, vol. 10, article 1645, 2019.

[14] J. J. Galipeau and L. L. Sensébé, "Mesenchymal stromal cells: clinical challenges and therapeutic opportunities," Cell Stem Cell, vol. 22, no. 6, pp. 824-833, 2018. 
[15] M. Kabat, I. I. Bobkov, S. Kumar, and M. Grumet, "Trends in mesenchymal stem cell clinical trials 2004-2018: is efficacy optimal in a narrow dose range?," Stem Cells Translational Medicine, vol. 9, no. 1, pp. 17-27, 2020.

[16] Z. Cesarz and K. Tamama, "Spheroid culture of mesenchymal stem cells," Stem Cells International, vol. 2016, Article ID 9176357, 11 pages, 2016.

[17] M. W. Laschke and M. D. Menger, "Life is 3D: boosting spheroid function for tissue engineering," Trends in Biotechnology, vol. 35, no. 2, pp. 133-144, 2017.

[18] N. Cheng, S. Chen, J. Li, and T. Young, "Short-term spheroid formation enhances the regenerative capacity of adiposederived stem cells by promoting stemness, angiogenesis, and chemotaxis," Stem Cells Translational Medicine, vol. 2, no. 8, pp. 584-594, 2013.

[19] Z. Cesarz, J. L. Funnell, J. Guan, and K. Tamama, "Soft elasticity-associated signaling and bone morphogenic protein 2 are key regulators of mesenchymal stem cell spheroidal aggregates," Stem Cells and Development, vol. 25, no. 8, pp. 622-635, 2016.

[20] J. H. Ylöstalo, T. J. Bartosh, K. Coble, and D. J. Prockop, "Human mesenchymal stem/stromal cells cultured as spheroids are self-activated to produce prostaglandin E2 that directs stimulated macrophages into an anti-inflammatory phenotype," Stem Cells, vol. 30, no. 10, pp. 2283-2296, 2012.

[21] T. J. Bartosh, J. H. Ylostalo, A. Mohammadipoor et al., "Aggregation of human mesenchymal stromal cells (MSCs) into 3D spheroids enhances their antiinflammatory properties," Proceedings of the National Academy of Sciences of the United States of America, vol. 107, no. 31, pp. 1372413729, 2010.

[22] S. S. Ho, K. C. Murphy, B. Y. K. Binder, C. B. Vissers, and J. K. Leach, "Increased survival and function of mesenchymal stem cell spheroids entrapped in instructive alginate hydrogels," Stem Cells Translational Medicine, vol. 5, no. 6, pp. 773-781, 2014.

[23] Q. Zhang, A. L. Nguyen, S. Shi et al., "Three-dimensional spheroid culture of human gingiva-derived mesenchymal stem cells enhances mitigation of chemotherapy-induced oral mucositis," Stem Cells and Development, vol. 21, no. 6, pp. 937-947, 2012.

[24] H. Y. Yeh, B. H. Liu, M. Sieber, and S. H. Hsu, "Substratedependent gene regulation of self-assembled human MSC spheroids on chitosan membranes," BMC Genomics, vol. 15, no. 1, p. 10, 2014.

[25] C. Tsai, Y. Liu, X. Yuan, and T. Ma, "Compaction, fusion, and functional activation of three-dimensional human mesenchymal stem cell aggregate," Tissue Engineering. Part A, vol. 21, no. 9-10, pp. 1705-1719, 2015.

[26] L. Guo, Y. Zhou, S. Wang, and Y. Wu, "Epigenetic changes of mesenchymal stem cells in three-dimensional (3D) spheroids," Journal of Cellular and Molecular Medicine, vol. 18, no. 10, pp. 2009-2019, 2014.

[27] N. Komatsu, M. Kajiya, S. Motoike et al., “Type I collagen deposition via osteoinduction ameliorates YAP/TAZ activity in 3D floating culture clumps of mesenchymal stem cell/extracellular matrix complexes," Stem Cell Research \& Therapy, vol. 9, no. 1, p. 342, 2018.

[28] M. Moya and G. Halder, "Hippo-YAP/TAZ signalling in organ regeneration and regenerative medicine," Nature Reviews Molecular Cell Biology, vol. 20, no. 4, pp. 211-226, 2019.
[29] P. J. Amos, S. K. Kapur, P. C. Stapor et al., "Human adiposederived stromal cells accelerate diabetic wound healing: impact of cell formulation and delivery," Tissue Engineering Parts A, vol. 16, no. 5, pp. 1595-1606, 2010.

[30] R. Coyle, J. Yao, D. Richards, and Y. Mei, “The effects of metabolic substrate availability on human adipose-derived stem cell spheroid survival," Tissue Engineering Parts A, vol. 25, no. 7-8, pp. 620-631, 2019.

[31] T. J. Bartosh, J. H. Ylöstalo, N. Bazhanov, J. Kuhlman, and D. J. Prockop, "Dynamic compaction of human mesenchymal stem/precursor cells into spheres self-activates caspasedependent Ill signaling to enhance secretion of modulators of inflammation and immunity (PGE2, TSG6, and STC1)," Stem Cells, vol. 31, no. 11, pp. 2443-2456, 2013.

[32] T. J. Bartosh and J. H. Ylostalo, "Preparation of antiinflammatory mesenchymal stem/precursor cells (MSCs) through sphere formation using hanging-drop culture technique," Current Protocols in Stem Cell Biology, vol. 28, no. 1, pp. 2B.6.1-2B.6.23, 2014.

[33] S. H. Bhang, S. Lee, J. Shin, T. Lee, and B. Kim, "Transplantation of cord blood mesenchymal stem cells as spheroids enhances vascularization," Tissue Engineering Parts A, vol. 18, no. 19-20, pp. 2138-2147, 2012.

[34] B. Jiang, L. Yan, Z. Miao, E. Li, K. H. Wong, and R. Xu, "Spheroidal formation preserves human stem cells for prolonged time under ambient conditions for facile storage and transportation," Biomaterials, vol. 133, pp. 275-286, 2017.

[35] M. Kim, H. Yun, D. Y. Park, B. H. Choi, and B. H. Min, "Three-dimensional spheroid culture increases exosome secretion from mesenchymal stem cells," Tissue Engineering and Regenerative Medicine, vol. 15, no. 4, pp. 427-436, 2018.

[36] L. M. Lawrence, A. Cottrill, A. Valluri et al., "Minimally manipulative method for the expansion of human bone marrow mesenchymal stem cells to treat osseous defects," International Journal of Molecular Sciences, vol. 20, no. 3, p. 612, 2019.

[37] Y. Xu, T. Shi, A. Xu, and L. Zhang, "3D spheroid culture enhances survival and therapeutic capacities of MSCs injected into ischemic kidney," Journal of Cellular and Molecular Medicine, vol. 20, no. 7, pp. 1203-1213, 2016.

[38] J. H. Ylöstalo, T. J. Bartosh, A. Tiblow, and D. J. Prockop, "Unique characteristics of human mesenchymal stromal/progenitor cells pre- activated in 3-dimensional cultures under different conditions," Cytotherapy, vol. 16, no. 11, pp. 14861500, 2014.

[39] J. H. Ylöstalo, N. Bazhanov, A. Mohammadipoor, and T. J. Bartosh, "Production and administration of therapeutic mesenchymal stem/stromal cell (MSC) spheroids primed in 3-D cultures under xeno-free conditions," Journal of Visualized Experiments, vol. 121, no. 121, p. 13, 2017.

[40] M. H. G. Costa, T. C. McDevitt, J. M. S. Cabral, C. L. da Silva, and F. Castelo Ferreira, "Tridimensional configurations of human mesenchymal stem/stromal cells to enhance cell paracrine potential towards wound healing processes," Journal of Biotechnology, vol. 262, pp. 28-39, 2017.

[41] J. P. Miranda, S. P. Camões, M. M. Gaspar et al., "The secretome derived from 3D-cultured umbilical cord tissue MSCS counteracts manifestations typifying rheumatoid arthritis," Frontiers in Immunology, vol. 10, no. 18, p. 14, 2019.

[42] J. A. Zimmermann and T. C. Mcdevitt, "Pre-conditioning mesenchymal stromal cell spheroids for immunomodulatory paracrine factor secretion," Cytotherapy, vol. 16, no. 3, pp. 331-345, 2014. 
[43] J. A. Zimmermann, M. H. Hettiaratchi, and T. C. McDevitt, "Enhanced immunosuppression of T cells by sustained presentation of bioactive interferon- $\gamma$ within three-dimensional mesenchymal stem cell constructs," Stem Cells Translational Medicine, vol. 6, no. 1, pp. 223-237, 2017.

[44] E. Redondo-Castro, C. J. Cunningham, J. Miller, H. Brown, S. M. Allan, and E. Pinteaux, "Changes in the secretome of tri-dimensional spheroid-cultured human mesenchymal stem cells in vitro by interleukin-1 priming," Stem Cell Research \& Therapy, vol. 9, no. 1, p. 11, 2018.

[45] J. H. Lee, Y. Han, and S. H. Lee, "Long-duration threedimensional spheroid culture promotes angiogenic activities of adipose-derived mesenchymal stem cells," Biomolecules \& Therapeutics, vol. 24, no. 3, pp. 260-267, 2016.

[46] M. Oberringer, M. Bubel, M. Jennewein et al., "The role of adipose-derived stem cells in a self-organizing 3D model with regard to human soft tissue healing," Molecular and Cellular Biochemistry, vol. 445, no. 1-2, pp. 195-210, 2018.

[47] I. Park, P. Chung, J. C. Ahn, and A. Leproux, "Human adiposederived stem cell spheroid treated with photobiomodulation irradiation accelerates tissue regeneration in mouse model of skin flap ischemia," Lasers in Medical Science, vol. 32, no. 8, pp. 1737-1746, 2017.

[48] S. Alimperti, P. Lei, Y. Wen, J. Tian, A. M. Campbell, and S. T. Andreadis, "Serum-free spheroid suspension culture maintains mesenchymal stem cell proliferation and differentiation potential," Biotechnology Progress, vol. 30, no. 4, pp. 974-983, 2014.

[49] R. J. Cho, Y. Kim, J. Kim, and Y. Oh, "Human adipose-derived mesenchymal stem cell spheroids improve recovery in a mouse model of elastase-induced emphysema," BMB Reports, vol. 50, no. 2, pp. 79-84, 2017.

[50] Y. Li, G. Guo, L. Li et al., "Three-dimensional spheroid culture of human umbilical cord mesenchymal stem cells promotes cell yield and stemness maintenance," Cell and Tissue Research, vol. 360, no. 2, pp. 297-307, 2015.

[51] N. Cheng, S. Wang, and T. Young, "The influence of spheroid formation of human adipose-derived stem cells on chitosan films on stemness and differentiation capabilities," Biomaterials, vol. 33, no. 6, pp. 1748-1758, 2012.

[52] K. Mineda, J. Feng, H. Ishimine et al., "Therapeutic potential of human adipose-derived stem/stromal cell microspheroids prepared by three-dimensional culture in non-cross-linked hyaluronic acid gel," Stem Cells Translational Medicine, vol. 4, no. 12, pp. 1511-1522, 2015.

[53] R. Z. Lin and H. Y. Chang, "Recent advances in threedimensional multicellular spheroid culture for biomedical research," Biotechnology Journal, vol. 3, no. 9-10, pp. 11721184, 2008.

[54] Y. Petrenko, E. Syková, and S. Kubinová, “The therapeutic potential of threedimensional multipotent mesenchymal stromal cell spheroids," Stem Cell Research \& Therapy, vol. 8, no. 1, p. 94, 2017.

[55] F. Dazzi, J. M. van Laar, A. Cope, and A. Tyndall, "Cell therapy for autoimmune diseases," Arthritis Research \& Therapy, vol. 9, no. 2, p. 9, 2007.

[56] M. Krampera, L. Cosmi, R. Angeli et al., "Role for interferongamma in the immunomodulatory activity of human bone marrow mesenchymal stem cells," Stem Cells, vol. 24, no. 2, pp. 386-398, 2006.

[57] G. Ren, L. Zhang, X. Zhao et al., "Mesenchymal stem cellmediated immunosuppression occurs via concerted action of chemokines and nitric oxide," Cell Stem Cell, vol. 2, no. 2, pp. 141-150, 2008.

[58] M. Sarem, O. Otto, S. Tanaka, and V. P. Shastri, "Cell number in mesenchymal stem cell aggregates dictates cell stiffness and chondrogenesis," Stem Cell Research \& Therapy, vol. 10, no. 10, p. 18, 2019.

[59] W. Wagner, S. Bork, P. Horn et al., "Aging and replicative senescence have related effects on human stem and progenitor cells," PLoS One, vol. 4, no. 6, article e5846, 2009.

[60] C. D. Luzzani and S. G. Miriuka, "Pluripotent stem cells as a robust source of mesenchymal stem cells," Stem Cell Reviews and Reports, vol. 13, no. 1, pp. 68-78, 2017.

[61] J. C. Bloor, A. Patel, J. E. Griffin et al., "Production, safety and efficacy of iPSC-derived mesenchymal stromal cells in acute steroid-resistant graft versus host disease: a phase I, multicenter, open-label, dose-escalation study," Nature Medicine, vol. 26, no. 11, pp. 1720-1725, 2020.

[62] S. Eto, M. Goto, M. Soga et al., "Mesenchymal stem cells derived from human iPS cells via mesoderm and neuroepithelium have different features and therapeutic potentials," PLoS One, vol. 13, no. 7, article e0200790, 2018.

[63] Q. Lian, Y. Zhang, J. Zhang et al., "Functional mesenchymal stem cells derived from human induced pluripotent stem cells attenuate limb ischemia in mice," Circulation, vol. 121, no. 9, pp. 1113-1123, 2010.

[64] W. X. Gao, Y. Q. Sun, J. Shi et al., "Effects of mesenchymal stem cells from human induced pluripotent stem cells on differentiation, maturation, and function of dendritic cells," Stem Cell Research \& Therapy, vol. 8, no. 1, p. 48, 2017.

[65] Y. Q. Sun, Y. Zhang, X. Li et al., "Insensitivity of human iPS cells-derived mesenchymal stem cells to interferon- $\gamma$-induced hla expression potentiates repair efficiency of hind limb ischemia in immune humanized nod scid gamma mice," Stem Cells, vol. 33, no. 12, pp. 3452-3467, 2015.

[66] J. Zhang, Y. C. Chan, J. C. Ho, C. W. Siu, Q. Lian, and H. F. Tse, "Regulation of cell proliferation of human induced pluripotent stem cell-derived mesenchymal stem cells via ether-à-gogo 1 (hEAG1) potassium channel," American Journal of Physiology, vol. 303, no. 2, pp. C115-C125, 2012.

[67] Y. Ding, X. Liang, Y. Zhang et al., "Rap1 deficiency-provoked paracrine dysfunction impairs immunosuppressive potency of mesenchymal stem cells in allograft rejection of heart transplantation," Cell Death \& Disease, vol. 9, no. 3, p. 386, 2018. 